\title{
8. Evaluating data visualization: Broadening the measurements of success
}

\author{
Arran L. Ridley and Christopher Birchall
}

\begin{abstract}
This chapter investigates the evaluation of data visualizations using observational research in an award-winning design studio. It outlines some professional and commercial forces that are involved in the shaping of evaluative strategies and identifies differences in methods and forms of evaluation in projects with different aims and intended audiences. The research showed that alongside quantitative headline figures of consumption, such as audience reach and interaction, qualitative measures of audience experience-which consider the sociocultural context of consumption - were sometimes included in evaluation strategies, but this varied between projects depending on the level of access to, and knowledge about, the audience. This chapter highlights the importance of such measures, outlines attempts to develop them, and comments on the potential to do so.
\end{abstract}

Keywords: Evaluation; Data visualization; Sociocultural context; Audience; Design studio; Observation

\section{Introduction}

Data visualization plays an important role in the information environment, communicating complex messages through simplified yet powerful representations of otherwise opaque data. Data visualization is deployed in different contexts and in various settings, such as the representation of business information within or between companies, the delivery of

\footnotetext{
Engebretsen, M. and H. Kennedy (eds.), Data Visualization in Society. Amsterdam: Amsterdam University Press, 2020 DOI 10.5117/9789463722902_CHO8
} 
products and services to personal consumers, and the communication of news and information in the public sphere. Within this broad landscape, data visualization is deployed to achieve different aims and objectives and can be tailored to do so for specific audiences. In a business setting, for example, the audience may be trained to expect, interpret, and use data visualizations, in formats with which they become familiar, to meet the requirements of the business. In contrast, consumers within the wider public sphere are a diverse and much more unpredictable audience within which the skills and experience, time, and environment required to successfully navigate complex data visualizations cannot be known. Evaluation of data visualizations can, therefore, be complex when these products are designed to meet goals which exist on a spectrum from cognitive, affective, behavioural, and even physiological human responses on the one hand (Zube, Sell, \& Taylor, 1982), to the less personal modern commercial and communications imperatives, such as views, shares, click-throughs, and sales conversions, on the other. For this reason, strategies for evaluating data visualizations can include dimensions such as the metrics of reach, consumption, and audience interaction that are common in social media and web analytics (Aisch, 2017; Baur, 2017; Wattenberg, 2005) and the user testing and feedback of HCI (Human-Computer Interaction) and usability research (Freitas, Pimenta, \& Scapin, 2014; Vogel, Kurti, Milrad, \& Kerren, 2011). In some cases attempts may be made to measure understanding and impact generated by the visualization within audiences (Sheppard, 2005), and work such as the Seeing Data Project highlighted the potential for the collection of qualitative data from consumers to capture their opinions and feelings about a visualization (Kennedy \& Hill, 2017).

This chapter describes how different production and consumption contexts can combine to create the need for different evaluative practices in different situations, where data visualizations operate under different conditions, with different audiences, and with different aims and goals. It also illustrates how professional production processes can support and prioritize some of these evaluative practices more than others. The existence of conventions within data visualization production-discussed in detail in the literature (Barnhurst, 1994; Coopmans, Vertesi, Lynch, \& Woolgar, 2014; Few, 2012; Kennedy, Hill, Aiello, \& Allen, 2016; Kosara, 2007a; Tufte, 2001) - is part of a network of influences that help to shape the processes of data visualization practice. Here, these processes are described also as an influence on the set of values used to evaluate data visualizations, which may limit the methods and forms of evaluation used. These values are derived from factors which exist largely on the production or supply side 
of data visualization, and while there have been studies of conditions on the demand side (Kennedy et al., 2016) the factors affecting consumption of data visualizations, such as the sociocultural context of consumption, are less well documented or established. Audience preferences, abilities, and experiences are often difficult to measure, particularly in real time, and so evaluation methods often include assumptions about audiences rather than involving them in evaluative processes.

Through observational fieldwork within a leading data visualization production studio, this chapter illustrates how the sociocultural context of consumption may be considered during the evaluation of visualizations in some production pathways, particularly where that context is most readily accessible, but not in others. From this we build a broader argument about the potential improvements to evaluative practices that could be enabled by the consideration of sociocultural contexts of consumption within evaluation, which might enable us to better understand how the social context of the consumer can impact the reception, and perhaps inform the design, of visualizations that perform important functions in the public sphere. Although the chapter is based on a small-scale exploratory study on only one agency, we propose that it nonetheless provides some useful food for thought.

\section{Interrogating the sites of production}

The decisions about evaluative practices on the supply side in which practitioners, clients, and other interested parties judge a product according to their preferred success criteria are made by various people in various roles. At its simplest definition, a practitioner can be considered as any person who produces a data visualization, such as the freelancers or academics who might be involved in collecting and sorting data, selecting tools and chart types, and producing and deploying finished data visualizations to suit their needs. However, data visualizations are often produced in professional settings such as design studios, within which multiple actors are present, each with varying degrees of influence on the production process. These actors extend beyond those directly involved in the design process (such as visual designers, coders, or UX/UI designers) to include project managers, PR staff, upper management, and clients (a catch-all term that itself includes a complex network of actors and stakeholders who may not all share the same goal). Although they may come from different disciplinary backgrounds, data visualization practitioners are sometimes expected to have a skill set 
that encompasses everything from the ability to collect, scrape, and sort data, through to the production of a data visualization itself (Kirk, 2016). According to Kirk (2016), skills such as strong numeracy and a familiarity with basic statistics, as well as some knowledge of common spreadsheet software are prerequisites, as are a sense of curiosity and an openness to creativity regardless of previous experience or understanding of design guidelines. Data visualization practitioners can also be categorized by their primary skill sets: 'designers' whose main experience is in design contrast with 'programmers' who 'create visualizations and visualization tools programmatically' (Bigelow et al., 2014). Bigelow et al. (2014) claim that both, however, are thought to share expertise in data analysis.

The diverse skill sets and interdisciplinarity of data visualization practitioners are important influences in the process of turning data, through the steps of analytical abstraction, into visual representations. These influences may be acknowledged by practitioners through efforts to provide transparency and authenticity to datasets, through the inclusion of data sources, annotations, or corrections in an attempt to create 'data provenance' (D'Ignazio, 2015; Hullman \& Diakopoulos, 2011; Tufte, 2006). These practices - and the conventions that help to shape them-are part of the 'editorial layers' that shape visualizations (Hullman \& Diakopoulos, 2011), but they can also impact decisions made about evaluation by influencing the values and priorities that are the focus of evaluation efforts. The production environment and processes are, therefore, an important factor in any investigation into the evaluation of data visualizations, and it is pertinent to include the site of data visualization production - the people, roles, relationships, rules and conventions, aims, goals, and pressures -in this analysis. As well as questioning the 'material economy behind the data' (D'Ignazio, 2015), investigating the site of data visualization production can make visible the role of different actors present and make it possible to ask questions about what influence each stakeholder has on evaluative practices and under what conditions these influences might be exerted.

The empirical evidence presented here was collected over four weeks on-site at an award-winning commercial design studio which specializes in data visualization. The studio produces data-driven products, such as business dashboards and static or interactive data visualizations for print and digital. They have around 30 employees, including permanent and temporary staff such as freelancers and interns, and serve domestic and international clients of varying size. Participant observation was undertaken to gain an understanding of production processes at the studio and to analyse the relationship of these with the design and implementation of product 
evaluation. Two live projects were followed throughout the period of observation. First, a large-scale production of a business dashboard, being designed to streamline the existing process of producing reports within a client's corporation. Second, a set of data visualizations intended to form the basis of a style guide to be utilized by a client. Alongside these live projects, data were collected about several completed projects of which one-a healthcare app designed to aid in the recovery of cancer patients-will be discussed in this chapter. During observation of the live projects, documentation of meetings and interviews with the project team members were undertaken, as well as analysis of the supporting documents produced. For the completed projects, interviews were conducted alongside analysis of the archived documentation of the project process.

\section{Considering evaluation methods}

The arguments of the previous sections have posited that design conventions and assumed best practices amongst communities of practitioners shape visualizations. Data visualizations are produced according to different aims and strategies and thus the mode of evaluation utilized in each case would be expected to vary accordingly to the goal of the producer. It is widely accepted that the general goal of data visualization is to visually communicate non-visual data (Kosara, 2007; Manovich, 2011; Munzner, 2014), but this oversimplifies the different goals of practitioners, who are working within different industries or disciplines and therefore have differing measures of success. A data visualization can be designed to be a clear communication of data for business purposes, a powerful political message, an emotive headline to attract eyeballs to a particular news item, or a beautiful artefact garnering attention in its own right (Rost, 2017).

Evaluating visualizations according to these different goals is challenging and some success criteria are easier to measure than others. For example, counting how many people have seen a visualization might be an appropriate and attainable evaluation strategy if the goal is to achieve a wide circulation, but measuring an emotional response to a data visualization, if such an outcome was the project goal, requires a much more complex evaluation method. Often the most accessible method available is selected and web and social media analytics provide convenient quantitative methods of evaluation. These popular approaches to evaluation are attractive to the producers of data visualization within a commercial design studio as they measure some dimensions of consumption that translate into commercial 
success. While mindful of the range of qualities required in the finished artefact, practitioners must consider how these artefacts can be utilized to generate more commissions. Popular measures such as shares or likes are a means of signalling the potential to reach prospective customers. One such analytics-based evaluation examining a visualization on the New York Times website found that only 10-15 percent of all visitors to the page clicked on the visualization, and concluded that this interactive graphic was therefore a 'waste of time and money' (Baur, 2017). The impact of the visualization on the consumer is not measured through this metric, however; any increase in knowledge or other impact on the consumer is not known. Herein lies the issue with employing numeric measures of consumption as a means of evaluation: it might capture the metrics of 'engagement', the amount of times something has been interacted with, shared, or viewed, but it doesn't capture why this is happening. Engagement with the process of consumption can help to answer these more difficult questions.

Evaluation is not only done after distribution of a final product. It can also take place at various places along the development process, at the predesign, design, prototype, deployment, or redesign stage (Lam et al., 2012). User testing is a common form of evaluation within design processes, which can be utilized in different ways. As part of academic research, it often takes place within laboratory settings and deals with specific elements such as memorability (Borkin et al., 2013), speed of task completion or recall (Chin et al., 2009), or the effectiveness of particular visual elements (Skau \& Kosara, 2016). When combined with measurement of participant satisfaction, such studies sometimes aim to judge whether certain visualization techniques are more or less effective than others for representing and communicating data (Chin et al., 2009; Haroz \& Whitney, 2012). The focus of such approaches to user testing is often on the data visualization itself, the effectiveness of the visual elements, and presentation styles. These are important factors in the design of visualizations, but so is their consumption. Within the controlled conditions of the lab, such tests can evaluate certain elements of data visualizations, but they do not take into account the conditions of consumption in 'the wild'. Outside of the lab, where consumers encounter media products in diverse, often unpredictable situations, the impact of visualizations may vary as consumers experiment with or appropriate products, devoting different amounts of attention, or utilizing different emotional or cognitive processes (Oudshoorn \& Pinch, 2003). As Kennedy, Hill, Allen, and Kirk (2016) argue 'who users are, contexts of visualization use and other factors outside of the visualization text are also important in determining visualization effectiveness'. Some such studies, situated in 
the field of Human Computer Interaction and UX/UI design, do not provide much information about the participants themselves, even in studies with low numbers of participants, as Kennedy, Hill, Allen, and Kirk (2016) note. Participants in these studies are rarely representative of the general public or even indeed the intended audience, reflecting the emphasis on putting new features or chart types to the test.

\section{Observations of evaluative practices within a design studio}

Three cases examined during fieldwork, including the two live projects and one completed project, were designed with different goals and with different audiences in mind. These differences led to different processes being utilized during production and these differences were mirrored in the evaluation strategies used for each project.

The first live project studied — the business dashboards—involved a product used by the client to analyse data and to inform decisions. This was produced for a specific business context, defined by business processes and needs, which determined specific, measurable success criteria. As the product was used by its employees, the client brought an understanding of the environment and conditions in which the product was consumed. Design studio staff working on the project made considerable efforts to understand the end users themselves through interviews and workshops, to extend the knowledge brought by the client. Participants at these workshops were asked to detail their thoughts and feelings in relation to their current workflow, which helped the practitioners to understand the key moments and actors in the decision-making process and where within this process the tool would sit. Consistent contact with the client was maintained through an iterative development and evaluation process, in which versions of the tool were released to the client who in turn tested them and fed back to the practitioners, who then amended the product and initiated further cycles of this process until the tool was considered complete. In addition to this testing carried out with the client, further user testing was undertaken internally with other practitioners not attached to the project, in which novel or new functionality or features were tested or 'validated' before being revealed to the client. This process allowed for a great deal of information to be gathered about the sociocultural context of consumption of the visualization within the client organization, within an evaluation method embedded within the production process.

In one archived project that was observed, which involved the design of visualizations within a mobile app to be used by survivors of cancer, the 
sociocultural context of consumption was also considered during design. This time, however, this inclusion was more limited, reliant upon the expertise of the client rather than user testing and iterative design. The app was designed to provide personalized information to help users to respond to the demands of the illness and its treatment and to aid recovery by relaying custom instructions for the user to follow. The context of consumption was therefore quite specific, and the client was able to relay clear expectations of the desired outcome to the practitioners based on detailed knowledge of the consumer. However, unlike the controlled setting of the corporate office in which the business dashboards would perform, these visualizations were likely to be consumed in a wide variety of contexts by consumers who might share the common experience of being cancer survivors but have different preferences, capabilities, contexts, and experiences of consumption. The potential users of an app like this are harder to recruit for user testing, due to the very specific nature of the audience, and their condition being sensitive in nature. In this case, knowledge of the sociocultural context of consumption was provided by the expert client. Where this project differs from the business dashboard project is in the greater potential scope of consumption contexts and the limited access to the consumer. In these situations, practitioners made use of other available resources for user testing (studio staff members, for example); a practice not unusual in the design community (Dickey-Kurdziolek, 2018).

The second live project observed during the research period was the design of a style guide for a client and this project involved far less engagement with the sociocultural context of consumption. Rather than producing public-facing visualizations directly, this product was designed to enable their production by the client themselves, who could utilize different datasets to produce visualizations for different audiences, in accordance to the design guidelines laid out in the style guide. These guidelines were produced using 'fake' or 'dummy' data as placeholders for future content, in contrast to the business dashboard which involved a lengthy investigation into the properties of the available data before design. The design and evaluation process of this project relied upon assumptions about data visualization best practice rather than on considerations relating to the end users of the client's visualizations.

The three projects discussed here show how different production scenarios can influence evaluation strategies and specifically how the sociocultural context of consumption may or may not be included within them. The business dashboards project involved a consumption context that was known and defined, through business logics, expertise, and testing 
carried out by the client and the practitioners. This context could therefore be incorporated into comprehensive evaluative strategies within an iterative development process. The app for cancer sufferers involved descriptions of potential sociocultural context of consumption, provided by the client. However, while the client was able to detail some specific information about the potential end user, the consumers themselves - and their personal consumption environments and situations - remained out of reach for the practitioner. Finally, the style guide was designed to direct future visualization production by the client and as such was produced without knowledge of either the datasets to be included or the audiences to be reached. In this case, evaluation incorporating the client's consumers and their context of consumption was unlikely, with assurances of quality instead provided by the assumed best practices embedded in the style guide.

\section{Evaluating the sociocultural context of consumption}

Visualizations are often produced and consumed in diverse environments, and both the processes of encoding, during production, and decoding, during consumption, are subject to the 'sociocultural milieu' of the producer and consumer (Hall, 1973). These diverse environments could possibly lead to unpredictable consumption effects, and may result in the data visualization being decoded by the consumer in ways that may be unexpected by the producer. The consumer undertakes a complex process of decoding through cultural, perceptual, cognitive, and psychological lenses to extract meaning (Hullman \& Diakopoulos, 2011). Educational and class background can be one influence on this (Bourdieu, 2010; Hall, 1973); the role of emotions in engagement with media artefacts, particularly when content matter is emotive, may be another (Hakone et al., 2017). The examples discussed above illustrate how this decoding process is evaluated to different extents, and that the sociocultural context of consumption can be given greater or lesser attention during evaluation in different projects.

As discussed earlier, consumption of online visualizations can be measured in digital spaces in terms of audience reach and interaction through popular web and social media analytics, but metrics relating to the impact of visualizations on individuals are harder to come by. How does one measure the amount of knowledge gained from a visualization, or if the knowledge taken away is what the producer intended? How can the emotional impact of visualizations be determined? Moreover, other factors relating to consumption, such as whether the consumer is alone or with 
others, concentrating on a subject or viewing casually, technological devices used, or viewing environments (such as at home, on the bus or in a pub), are rarely considered and difficult to capture. These factors - the sociocultural context of consumption-provide a significant challenge to efforts to build comprehensive evaluation methods for data visualizations, and therefore to our ability to build a complete picture of their consumption and impact.

The Seeing Data project shed light on this issue, identifying six factors which could impact engagement with data visualizations: subject matter; source/media; beliefs and opinions; time; emotions; and confidence and skills (Kennedy et al., 2016). Echoing aspects from media effects theory, these factors can have an impact on various groups in society and on individual media users (Potter, 2012; Valkenburg, Peter, \& Walther, 2016). Amongst the findings of the Seeing Data study were clear examples of how engagement with visualizations varied according to factors other than the design decisions made during production. Consumers engaged more closely with visualizations which focused on one of their pre-existing interests; visualizations from particular sources were judged to be more or less trustworthy; enjoyment of visualizations varied depending upon whether preformed opinions were challenged (Kennedy et al., 2016). The sociocultural context of consumption is therefore an active factor influencing impact and must be taken into consideration within the evaluative process. Beyond the best practice principles of professional designers is the messy social world where consumption takes place and a myriad of personal and cultural influences impact engagement, enjoyment, and comprehension.

While rare in practice, opportunities for sociocultural evaluation do exist. The Seeing Data project experimented with a 'widget' (2017) within data visualizations that allowed consumers to submit emotional or cognitive feedback about their experiences. Contemporary digital communications technologies offer potential for more evaluation like this, with possibilities for increasing the scale of such studies offered by technical solutions such as browser plug-ins and mobile apps that allow feedback to be gathered on visualizations as they are encountered in everyday digital consumption. Like the 'widget' of the Seeing Data project, these technologies provide the potential for consumers to participate actively in evaluation, reporting on their experiences and reactions, understanding, or other dimensions of impact, offering the possibility of richer, deeper evaluation which considers the personalized environments of consumption and the effects of visualization upon individuals, rather than simply the rate of consumption in general. Of course, within any attempt to produce more context-specific methods of evaluation, the communicative strategies 
and aims of production projects need to remain in focus, be considered, and matched to evaluation methods, so that the most suitable evaluative method, which meets both the aims of producers and the context of consumers, can be implemented.

\section{Conclusion}

Different project goals can be associated with different success criteria, and these can lead to different evaluative methods being employed. These goals and success criteria are likely to be focused on supply-side demands, such as audience size or interactions, and less often engage with the demand side - the sociocultural context of consumption. Observational fieldwork in a data visualization studio suggested that the most quantitative and simplest measurements of consumption through popular analytics methods were employed where client or project goals gave them value, and the sociocultural context was only embedded within evaluative methods where resources and expertise allowed, and where commercial imperatives made it appropriate and gave it value, too. Where intended audiences were known and definable, and where the sociocultural context of consumption was relatively well understood, efforts to incorporate this context into both production and evaluation could be comprehensive. However, where data visualizations were aimed at audiences that were more diverse and less well understood, the sociocultural context of consumption was much less likely to feature in evaluation. The measurement of the diverse consumption contexts present in the public sphere is of course difficult. It is within these public sphere environments, though, that sociocultural context is at its most variable and likely to assert its greatest effect. Data visualizations are produced to serve many purposes, but by making information accessible and available in the public sphere they can have implications for democratic functions such as decision-making and preference formation by citizens. Of course, in a commercial production environment the needs of the practitioner and clients must be acknowledged, and context-specific evaluation needs to be considered in relation to, rather than in place of, existing evaluative methods. If some of the affordances of digital media-in apps, websites, and browsers - can be utilized to incorporate sociocultural evaluation into existing evaluative practices, however, a richer and more rounded form of evaluation could be developed that includes measurements of impact and experience alongside the quantitative metrics that exist already. 


\section{References}

Aisch, G. (2017). In defense of interactive graphics. Vis4. Retrieved November 2, 2017 from https://www.vis4.net/blog/2017/03/in-defense-of-interactive-graphics/

Barnhurst, K. G. (1994). Seeing the newspaper. New York: St. Martin's Press.

Baur, D. (2017, March 13). The death of interactive infographics? Medium. Retrieved from https://medium.com/@dominikus/the-end-of-interactive-visualizations$525^{8} 5$ dcafcb

Bigelow, A., Drucker, S., Fisher, D., \& Meyer, M. (2014). Reflections on how designers design with data. Proceedings of the 2014 International Working Conference on Advanced Visual Interfaces-AVI ' '4. http://doi.org/10.1145/2598153.2598175

Borkin, M. A., Vo, A. A., Bylinskii, Z., Isola, P., Sunkavalli, S., Oliva, A., \& Pfister, H. (2013). What makes a visualization memorable? IEEE Transactions on Visualization and Computer Graphics, 19(12), 2306-2315. http://doi.org/10.1109/ TVCG.2013.234

Bourdieu, P. (2010). Distinction: A social critique of the judgement of taste. (R. Nice Trans.). London: Routledge.

Chin, G., Singhal, M., Nakamura, G., Gurumoorthi, V., \& Freeman-Cadoret, N. (2009). Visual analysis of dynamic data streams. Information Visualization, 8(3), 212-229. http://doi.org/10.1057/ivs.2009.18

Coopmans, C., Vertesi, J., Lynch, M., \& Woolgar, S. (2014). Introduction: Representation in scientific practice revisited. In: C. Coopmans, J. Vertesi, M. Lynch, \& S. Woolgar (Eds.), Representation in scientific practice revisited. (pp. 15-36). Cambridge, MA: MIT Press.

D'Ignazio, C. (2015). What would feminist data visualization look like? Retrieved September 5, 2018 from https:/civic.mit.edu/2015/12/01/feminist-data-visualization/

Dickey-Kurdziolek, M. (2018, January 4). Discovery on a Budget: Part I. Retrieved from https://alistapart.com/article/discovery-on-a-budget-part-i

Few, S. (2012). Show me the numbers: Designing tables and graphs to enlighten (2nd ed.). Burlingame, CA: Analytics Press.

Freitas, C. M. D. S., Pimenta, M. S., \& Scapin, D. L. (2014). User-centered evaluation of information visualization techniques: Making the HCI-InfoVis connection explicit. In: W. Huang (Ed.), Handbook of human centric visualization. (pp. 315336). New York, NY: Springer. http://doi.org/10.1007/978-1-4614-7485-2_12

Hakone, A., Harrison, L., Ottley, A., Winters, N., Gutheil, C., Han, P. K. J., \& Chang, R. (2017). PROACT: Iterative design of a patient-centered visualization for effective prostate cancer health risk communication. IEEE Transactions on Visualization and Computer Graphics, 23(1), 601-610. http://doi.org/10.1109/TVCG.2016.2598588

Hall, S. (1973). Encoding and decoding in the television discourse. Birmingham: Centre for Cultural Studies, University of Birmingham. 
Haroz, S., \& Whitney, D. (2012). How capacity limits of attention influence information visualization effectiveness. IEEE Transactions on Visualization and Computer Graphics, 18(12), 2402-2410. http://doi.org/10.1109/TVCG.2012.233

Hullman, J., \& Diakopoulos, N. (2011). Visualization rhetoric: Framing effects in narrative visualization. IEEE Transactions on Visualization and Computer Graphics, 17(12), 2231-2240. http://doi.org/10.1109/TVCG.2011.255

Kennedy, H., \& Hill, R. L. (2017). The feeling of numbers: Emotions in everyday engagements with data and their visualisation. Sociology, 52(4), 830-848. https:// doi.org/10.1177/0038038516674675

Kennedy, H., Hill, R. L., Aiello, G., \& Allen, W. (2016). The work that visualisation conventions do. Information, Communication and Society, 19(6), 715-735. https:// doi.org/10.1080/1369118X.2016.1153126

Kennedy, H., Hill, R. L., Allen, W., \& Kirk, A. (2016). Engaging with (big) data visualizations: Factors that affect engagement and resulting new definitions of effectiveness. First Monday, 21(11). https://doi.org/10.5210/fm.v21111.6389

Kirk, A. (2016). Data visualisation: A handbookfor data driven design. London: Sage. Kosara, R. (2007a). A Tale of Two Types of Visualization and Much Confusion. Retrieved June 22, 2017 from https://eagereyes.org/criticism/tale-of-two-types Kosara, R. (2007b). A Tale of Two Types of Visualization and Much Confusion. Retrieved June 22, 2017 from https://eagereyes.org/criticism/tale-of-two-types Lam, H., Bertini, E., Isenberg, P., Plaisant, C., \& Carpendale, S. (2012). Empirical studies in information visualization: Seven scenarios. IEEE Transactions on Visualization and Computer Graphics, 18(9), 1520-1536. http://doi.org/10.1109/ TVCG.2011.279

Manovich, L. (2011). What is visualisation? Visual Studies, 26(1), 36-49. https://doi. org/10.1080/1472586X.2011.548488

Munzner, T. (2014). Visualization analysis and design. Hoboken: CRC Press.

Oudshoorn, N., \& Pinch, T. (2003). How users matter: The co-construction of users and technologies. Cambridge, MA: MIT Press.

Potter, W. J. (2012). Media effects. Thousand Oaks, CA: Sage Publications.

Rost, L. C. (2017, March 10). Why Do We Visualise Data? [Blog post] Retrieved from https://lisacharlotterost.github.io/2017/o3/10/why-do-we-visualize-data/

Seeing Data. (2017). Rate these visualisations! Seeing Data. Retrieved November 17, 2017 from http://seeingdata.org/developing-visualisation-literacy/ rate-these-visualisations/

Sheppard, S. R. J. (2005). Landscape visualisation and climate change: The potential for influencing perceptions and behaviour. Environmental Science \& Policy, 8(6), 637-654. http://doi.org/10.1016/J.ENVSCI.2005.08.002

Skau, D., \& Kosara, R. (2016). Arcs, angles, or areas: Individual data encodings in pie and donut Charts. Computer Graphics Forum, 35(3), 121-130. 
Tufte, E. (2001). The visual display of quantitative information (2nd ed.). Cheschire, CT: Graphics Press.

Valkenburg, P. M., Peter, J., \& Walther, J. B. (2016). Media effects: Theory and research. Annual Review of Psychology, 67(1), 315-338. http://doi.org/10.1146/ annurev-psych-122414-033608

Vogel, B., Kurti, A., Milrad, M., \& Kerren, A. (2011). An interactive web-based visualization tool in action: User testing and usability aspects. In: 2011 IEEE nth International Conference on Computer and Information Technology. (pp. 403-408). IEEE. http://doi.org/10.1109/CIT.2011.68

Wattenberg, M. (2005). Baby names, visualization, and social data analysis. Proceedings of the 2005 IEEE Symposium on Information Visualization (INFOVIS'05), 1-7 http://doi.org/10.1109/INFVIS.2005.1532122

Zube, E. H., Sell, J. L., \& Taylor, J. G. (1982). Landscape percetion: Research, application and theory. Landscape Planning, 9(1), 1-33. https://doi.org/10.1016/o3043924(82)90009-0

\section{About the authors}

Arran L. Ridley is currently working on 'Seeing Data', a funded ESRC and White Rose DTC project that is investigating the possibilities of how the effectiveness of data visualization can be defined and measured and the potential for capturing and understanding people's engagement with data visualizations.

Chris Birchall is a Lecturer in Digital Media at the School of Media and Communication, University of Leeds. His research interests include digital methods and their application to the study and practice of digital citizenship and political communication online, and mobile and digital technologies, social change, and the human experience. 